\title{
Clasificación de los puertos españoles atendiendo a su tipología de tráfico e infraestructuras
}

\author{
CONCEPCIÓN CORTÉS RODRÍGUEZ ${ }^{a}$, ENCARNACIÓN CORDÓN LAGARES ${ }^{a}$, \\ ANA GONZÁLEZ GALÁN ${ }^{a}$, JUAN JOSÉ GARCíA DEL HOYO ${ }^{a}$
}

a Universidad de Huelva, Facultad de Ciencias Empresariales y Turismo, Campus de la Merced, Plaza de la Merced, 11, 21002 Huelva, España. E-mail: ccortes@uhu.es, ecordon@uhu.es, ana.gonzalez@dehie.uhu.es, hoyo@uhu.es

\begin{abstract}
RESUMEN
El principal objetivo de este trabajo es la clasificación de los puertos españoles de interés general utilizando diversos indicadores que caracterizan la actividad portuaria referente al transporte de mercancías y pasajeros e indicadores de infraestructuras y servicios portuarios. La información ha sido obtenida principalmente a partir de las estadísticas de Puertos del Estado y de las memorias anuales de las Autoridades Portuarias.

Mediante el uso de diferentes técnicas del análisis multivariante, se analiza un conjunto de variables cuantitativas y cualitativas. El Análisis Factorial de Componentes Principales ha permitido reducir el número de variables en cuatro factores, y con el Análisis de Conglomerados se obtuvo la clasificación de los puertos.

Como conclusiones relevantes del análisis, cabe resaltar la existencia de tres grupos de puertos de titularidad estatal claramente diferenciados en función de su nivel de especialización en el tráfico portuario e infraestructuras, y otro conjunto de puertos que destacan por el tráfico de pasajeros de cruceros.

Palabras clave: Análisis multivariante, Puertos, Tráfico, Infraestructuras.
\end{abstract}

\section{Classification of Spanish Ports According to Their Type of Traffic and Infrastructures}

\begin{abstract}
The main purpose of this paper is the classification of the Spanish ports of general interest using a set of indicators related to port activity such transport of goods and passengers, infrastructures, and port services. The information has been mainly obtained from the State Port statistics and the annual reports of Port Authorities.

We analyse a set of quantitative and qualitative variables through different multivariate analysis techniques. Principal Components Analysis allowed us to reduce the number of variables in four factors, and the classification of the ports has been carried out through Cluster Analysis.

The main finding of this paper reveal the existence of clearly different three groups of the state ports depending on their level of specialization in port traffic port and infrastructures, and other ports group that stand out for their cruise passenger traffic.
\end{abstract}

Keywords: Multivariate analysis, Ports, Traffic, Infrastructures.

Clasificación JEL: C38, L91

Artículo recibido en junio de 2018 y aceptado en septiembre de 2018

Artículo disponible en versión electrónica en la página www.revista-eea.net, ref. ə-36311

ISSN 1697-5731 (online) - ISSN 1133-3197 (print) 


\section{INTRODUCCIÓN}

España es el país de la Unión Europea que cuenta con mayor longitud de costa, $7.879 \mathrm{~km}$, lo que junto a su privilegiada situación geográfica, próxima al eje de las rutas marítimas más importantes del mundo, favorece el transporte marítimo de mercancías y el comercio internacional con otros países ${ }^{1}$. El enclave estratégico del Estrecho de Gibraltar (entre el océano Atlántico y el mar Mediterráneo) lo convierten en el segundo más transitado del mundo (después del Canal de la Mancha). Según datos de la Organización Mundial del Comercio (OMC, 2018), el 80\% del total de mercancías que se mueve en el mundo se realiza mediante el transporte marítimo. En España más del 90\% de nuestras fronteras son marítimas. Esto coloca a los puertos españoles como la primera y última parada de las principales compañías navieras que operan entre América, el sur de Europa, el Extremo Oriente y también el continente africano.

Desde la década de los 60, el tráfico portuario en el conjunto portuario no ha dejado de crecer, a excepción de los años 2008 y 2009 durante la crisis económica, alcanzándose la cifra record histórica de casi 545 Mill. de Tm. en 2017, un 5,96\% más que en 2016, según datos oficiales de Puertos del Estado.

La creciente evolución del tráfico de mercancías en los puertos españoles queda reflejada en el índice de conectividad del transporte marítimo. España ha mejorado un $21 \%$ su posición con respecto al acceso a mercados extranjeros en los últimos años. En el índice de conectividad de 2017, elaborado por la Agencia de Naciones Unidas para el Comercio y el Desarrollo (UNCTAD, 2017), ocupa el undécimo lugar en el ranking mundial y el cuarto de Europa.

El concepto de puerto ha sufrido una importante transformación desde su consideración como un paraje de la costa más o menos abrigado donde los buques quedaban al refugio de los peligros del mar y en el cual se permitía el tráfico marítimo (Art.13 de la Ley de Puertos de 7 de mayo de 1880).

La definición de puerto en la Ley 27/1992 de Puertos del Estado y de la Marina Mercante (LPEMM), mantenida por el Texto Refundido de la Ley de Puertos del Estado y de la Marina Mercante de 2011 (TRLPEMM), aparece recogida en su artículo 2 como el conjunto de espacios terrestres, aguas marítimas, e instalaciones que, situados en la ribera del mar o de las rías, reúnan condiciones físicas, naturales o artificiales, y de organización que permitan la realización de operaciones de tráfico portuario y sea autorizado para el desarrollo de estas actividades por la Administración pública competente. Además, resulta imprescindible la existencia de infraestructuras portuarias para poder efectuar las operaciones de tráfico.

\footnotetext{
${ }^{1}$ Según datos publicados por el Instituto Geográfico Nacional: Costas Peninsulares, $4.830 \mathrm{~km}$; Islas Baleares, 1.428 km; Canarias, 1.583 km; y Ceuta y Melilla, 38 km (IGN, 2018).
} 
Más allá de esta definición, el puerto moderno no es solo una cadena de transporte, sino que se concibe como nodo en la cadena logística y de transporte. De hecho, los puertos son esenciales tanto desde el punto de vista del comercio como del transporte, y en los tiempos actuales se constituyen en verdaderos puntos de distribución de las mercancías y puntos de conexión de la intermodalidad del transporte.

Las actividades portuarias relacionadas con el transporte de mercancías, buques, pasajeros y vehículos, entre otras, contribuyen directamente al empleo, a la inversión interior y al crecimiento del PIB del total de la actividad económica. En total, los puertos españoles generan aproximadamente el 1,1\% del PIB español y una tasa de empleo del 1,23\%. Además, la actividad portuaria aporta a España un valor añadido bruto anual superior a los 10.700 Mill. €. Por nuestros puertos pasan el $60 \%$ de las exportaciones y el $85 \%$ de las importaciones, lo que representa el 53\% del comercio exterior español con la Unión Europea y el 96\% con terceros países (Puertos del Estado, 2018).

Asimismo, España es la tercera potencia europea (por detrás de Alemania y Holanda) en movimiento portuario de contenedores, y la undécima a nivel mundial. Tres de nuestros puertos se sitúan entre los 15 más importantes del continente europeo en cuanto al tráfico de mercancías en contenedor. Según los datos de tráfico de contenedores del portal de estudios económicos PortEconomics (2018), el puerto de Valencia ocupa la quinta posición (4,83 Mill. de TEUs ${ }^{2}$ ), el de Algeciras la sexta (4,38 Mill. de TEUs) y el de Barcelona la décima (2,96 Mill. de TEUs), siendo este último el que registró mayor crecimiento de todos en el año 2017.

Por otra parte, el papel que desempeñan los puertos españoles en el tráfico de cruceros es muy relevante. España se ha convertido en el segundo destino de Europa, solo por detrás de Italia, alcanzando los 9 Mill. de pasajeros de cruceros en 2017, según datos oficiales. Los destinos españoles se han convertido en habituales de todas las rutas que cubren los distintos circuitos europeos, situándose algunos de nuestros puertos entre los mejores destinos mundiales. Los puertos de Barcelona y de las Islas Baleares en el Mediterráneo y los puertos de las Palmas y de Santa Cruz de Tenerife en el Atlántico son los que mueven más cruceristas al año.

Pocos trabajos se han encontrado en la literatura sobre la clasificación del sistema portuario español. García del Hoyo et al. (2001) analizan la interrelación existente entre las infraestructuras disponibles en tierra en cada uno de los puertos del litoral andaluz y la estructura del sector pesquero con base en los mismos. Por otra parte, Camarero et al. (2016) clasifican los puertos de interés general

\footnotetext{
${ }^{2}$ Es una medida estándar para contenedores de diferentes capacidades. Un TEU (Twenty Equivalent Unit) equivale a un contenedor ISO de 20 pies de longitud (6,1 m).
} 
mediante el análisis de conglomerados, a través de una serie de indicadores físicos y de explotación que caracterizan la actividad portuaria. En otro trabajo de Camarero y Camarero (2016) se utilizan indicadores físicos y de explotación junto con indicadores de sostenibilidad medioambientales, elegidos mediante un análisis clúster y otro de regresión, para la clasificación de Puerto Verde.

De este modo, el objetivo de este trabajo es realizar una clasificación de los puertos españoles de interés general agrupados en Autoridades Portuarias mediante dos técnicas del análisis multivariante: el Análisis Factorial y el Análisis de Conglomerados. Hasta ahora, un gran número de estudios portuarios en los últimos años se han centrado en el análisis estratégico de los puertos mediante su productividad y eficiencia (Wanhill, 1974; Baños-Pino et al., 1999; González y Trujillo, 2003, 2009). Así, en el trabajo de Wanhill (1974) se diseña un modelo para determinar el número de atraques óptimos. Por su parte, González y Trujillo (2003) analizan la eficiencia del sistema portuario español de titularidad estatal en relación al servicio de infraestructuras. Algunos autores como González Laxe et al. (2015) realizan un estudio que analiza el impacto de los factores que afectan a la selección portuaria en el sistema español mediante un Análisis Factorial Exploratorio.

El resto del estudio se ha estructurado del siguiente modo. En la Sección 2 se describe el actual sistema portuario español. En la Sección 3 se detallan las fuentes de datos empleados y se expone la metodología utilizada en la que se apoya el análisis empírico. En la Sección 4 se muestran los principales resultados obtenidos. Finalmente, en la Sección 5 se recogen las principales conclusiones de este trabajo.

\section{EL SISTEMA PORTUARIO ESPAÑOL EN 2017}

El sistema portuario español de titularidad estatal actual está regulado por el Texto Refundido de la Ley de Puertos del Estado y de la Marina Mercante (TRLPEMM) aprobado por el Real Decreto Legislativo 2/2011, de 5 de septiembre, que sustituye a la Ley 27/1992 y a sus posteriores modificaciones legislativas de 1997, 2003 y 2010.

La distinción entre puertos de interés general (de primer y segundo orden) y puertos de interés local (provinciales y municipales) aparece contemplada por primera vez en la Ley de Puertos de 1880 (Art. 15). En el Real Decreto de 17 de diciembre de 1851 se recoge por primera vez la titularidad estatal de algunos puertos españoles dependientes del entonces Ministerio de Fomento (Art. 1).

La Ley 27/1992, de 24 de noviembre, de Puertos del Estado y de Marina Mercante (LPEMM) sentó las bases esenciales sobre las que se configura el Sistema Portuario de hoy en día. La entrada en vigor de dicha Ley mantuvo la titularidad estatal de los puertos de interés general que ya subsistía desde el modelo de gestión establecido en la Constitución Española de 1978 (Art. 
149,1.20). Además, creó el Ente Público Puertos del Estado y, por último, dispuso un único modelo de gestión portuaria basado en unas entidades de derecho públicas denominadas Autoridades Portuarias.

El artículo 24 del TRLPEMM define las Autoridades Portuarias como organismos públicos con personalidad jurídica y patrimonio propios, así como plena capacidad de obrar. Están adscritas al Ministerio de Fomento a través de Puertos del Estado y se autofinancian, entre otras, a través de las tasas y tarifas. En cuanto a sus actividades, las Autoridades Portuarias se ocupan de la provisión, planificación y gestión de las infraestructuras y espacios portuarios, y del marco regulatorio de los servicios portuarios cuya prestación corresponde al sector privado.

En la actualidad, el sistema portuario español de titularidad estatal está constituido por 46 puertos con calificación de interés general gestionados por un total de 28 Autoridades Portuarias, cuya coordinación y control de eficiencia corresponde al Organismo Público Puertos del Estado, órgano también dependiente del Ministerio de Fomento.

A través de Puertos del Estado el gobierno ejecuta su política portuaria, en especial la coordinación y control de eficacia del sistema portuario. Así, las Comunidades Autónomas tienen competencias de ejecución sobre los puertos de interés general cuando el Estado no se reserve su gestión directa.

Tabla 1

Autoridades Portuarias españolas por Comunidad Autónoma.

\begin{tabular}{|l|l|}
\hline \multicolumn{1}{|c|}{ Comunidad Autónoma } & \multicolumn{1}{c|}{ Autoridades Portuarias } \\
\hline Andalucía & $\begin{array}{l}\text { Almería, Bahía de Algeciras, Bahía de Cádiz, Huelva, Málaga, } \\
\text { Motril, Sevilla }\end{array}$ \\
\hline Canarias & Las Palmas, Santa Cruz de Tenerife \\
\hline Cantabria & Santander \\
\hline Cataluña & Barcelona, Tarragona \\
\hline Ciudades Autónomas de Ceuta y Melilla & Ceuta, Melilla \\
\hline Comunidad Valenciana & Alicante, Castellón, Valencia \\
\hline Galicia & $\begin{array}{l}\text { A Coruña, Ferrol-San Cibrao, Marín y Ría de Pontevedra, Vigo, } \\
\text { Vilagarcía }\end{array}$ \\
\hline Islas Baleares & Baleares \\
\hline País Vasco & Bilbao, Pasaia \\
\hline Principado de Asturias & Avilés, Gijón \\
\hline Región de Murcia & Cartagena \\
\hline
\end{tabular}

Fuente: Elaboración propia a partir de datos de las estadísticas de Puertos del Estado y de las memorias anuales de las Autoridades Portuarias.

Los puertos de titularidad estatal actuales son los puertos de interés general que figuran en el listado del Anexo I del Real Decreto Legislativo 2/2011 del TRLPEMM (Tabla 2). 
Tabla 2

Puertos de interés general por Comunidad Autónoma.

\begin{tabular}{|l|l|}
\hline \multicolumn{1}{|c|}{ Comunidad Autónoma } & \multicolumn{1}{c|}{ Puertos de interés general } \\
\hline Andalucía & $\begin{array}{l}\text { Almería, Carboneras, Bahía de Algeciras, Tarifa, Cádiz y su bahía (que incluye } \\
\text { el Puerto de Santa María, el de la zona franca de Cádiz, Puerto Real, el Bajo } \\
\text { de la Cabezuela y Puerto Sherry), Huelva, Málaga, Motril, Sevilla y su ría }\end{array}$ \\
\hline Canarias & $\begin{array}{l}\text { Arrecife, Las Palmas (que incluye el de Salinetas y el de Arinaga), Guía de } \\
\text { Isora, Puerto Rosario, La Estaca, Los Cristianos, San Sebastián de la Gomera, } \\
\text { Santa Cruz de la Palma, Santa Cruz de Tenerife (que incluye el de Granadilla) }\end{array}$ \\
\hline Cantabria & Santander \\
\hline Cataluña & Barcelona, Tarragona \\
\hline Ciudades Autónomas de & Ceuta, Melilla \\
\hline Couta y Melilla & Alicante, Castellón, Gandía, Sagunto, Valencia \\
\hline Galicia & A Coruña, Ferrol y su ría, Marín y ría de Pontevedra, San Cibrao, Vigo y su ría, \\
\hline Islas Baleares & Vilagarcía de Arousa y su ría \\
\hline País Vasco & Bilbao, Pasaia \\
\hline Principado de Asturias & Avilés, Gijón-Musel \\
\hline Región de Murcia & Cartagena (que incluye la dársena de Escombreras) \\
\hline
\end{tabular}

Fuente: Elaboración propia a partir de datos de las estadísticas de Puertos del Estado y de las memorias anuales de las Autoridades Portuarias.

Para que un puerto marítimo sea declarado como puerto de interés general será suficiente que al puerto en cuestión le sea de aplicación alguna de las siguientes circunstancias (BOE 253, 20/10/2011, Art. 4 del Cap. 1 del TRLPEMM):

- Que se efectúen en ellos actividades comerciales marítimas internacionales (avituallamiento, reparación de los buques, operaciones de estiba desestiba, carga, descarga, almacenamiento de mercancías, etc., que justifiquen la utilización de medios mecánicos);

- Que su zona de influencia comercial afecte de forma relevante a más de una Comunidad Autónoma;

- Que sirvan a industrias o establecimientos de importancia estratégica para la economía nacional;

- Que el volumen anual y las características de sus actividades comerciales marítimas alcancen niveles suficientemente relevantes o respondan a necesidades esenciales de la actividad económica general del Estado;

- Que por sus especiales condiciones técnicas o geográficas constituyan elementos esenciales para la seguridad del tráfico marítimo (especialmente en territorios insulares).

De este modo, una misma Autoridad Portuaria puede llevar la administración, gestión y explotación de varios puertos de interés general. Esto ocurre con las dos Autoridades Portuarias canarias: Las Palmas, que gestiona actualmente los puertos de Arrecife, Rosario y Las Palmas (incluido el de Salineta y el de 
Arinaga), y la de Santa Cruz de Tenerife que gestiona los puertos de Guía de Isora, La Estaca, Los Cristianos, San Sebastián de la Gomera, Santa Cruz de la Palma y Santa Cruz de Tenerife (incluido el de Granadilla). Por otra parte, la Autoridad Portuaria de Valencia gestiona los puertos de Gandía, Sagunto y Valencia. Por último, en Andalucía, la Autoridad Portuaria de Bahía de Algeciras gestiona los puertos de Bahía de Algeciras y Tarifa; y la de Almería, los puertos de Almería y Carboneras.

En 2017 se movilizaron casi 16 Mill. de TEUs, un 10,5\% más en relación a 2016. Los puertos hubs de Valencia, Bahía de Algeciras y Barcelona representaron el 76,7\% del total de TEUs movidos, siendo el de Barcelona el que más creció en TEUs respecto al año anterior. En la Figura 1 que recoge el ranking de las 5 Autoridades Portuarias con un mayor tráfico de contenedores en España, se muestra que el puerto de Valencia pasó a ocupar la primera posición con 4,8 Mill. y un incremento de tan solo el 2,1\%, tras el drástico descenso del 7,9\% respecto al año anterior del puerto Bahía de Algeciras, que en 2017 se encuentra en segunda posición con 4,3 Mill., debido, entre otros factores, al conflicto de la estiba, que afectó de forma negativa a la instalación gaditana más que a otras españolas en el primer semestre del año. El puerto de Barcelona aumentó un 34,4\% pero mantiene su tercera posición con más de 3 Mill. de TEUs, seguido de los puertos de Las Palmas y Bilbao con cerca de 1,2 Mill. y 0,6 Mill., respectivamente, y con un incremento del $24,2 \%$ y $1,4 \%$, respectivamente.

Figura 1

Ranking de las 5 Autoridades Portuarias con mayor tráfico de contenedores en España (TEUs). Años 2016 y 2017

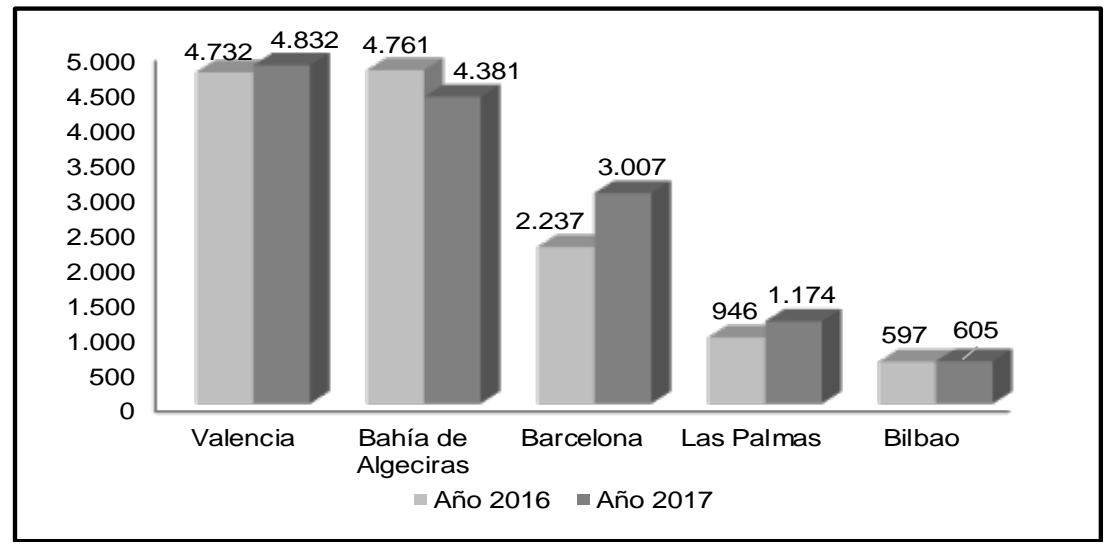

Fuente: Elaboración propia a partir de datos de las estadísticas de Puertos del Estado y de las memorias anuales de las Autoridades Portuarias.

El puerto Bahía de Algeciras es el que registró un mayor tráfico portuario de Tm. en España en el año 2017. Este puerto movió 101,5 Mill. de Tm., una cifra 
tan solo superada por los puertos europeos de Rotterdam, Amberes y Hamburgo. Le sigue en segunda posición el puerto de Valencia que alcanzó los 73,6 Mill. de Tm. y en tercera posición, el de Barcelona con 61,4 Mill. de Tm., siendo este último el que alcanzó un mayor crecimiento de todos respecto al año 2016 (un 26,3\%). En el otro extremo, y con menor tráfico portuario, se situó el puerto de Motril con 2,3 Mill. de Tm., seguido del de Villa García y el de Melilla con 1,2 Mill. de Tm. (Figura 2).

Figura 2

Ranking del tráfico portuario de las 28 Autoridades Portuarias (en Mill. de Tm.). Año 2017.

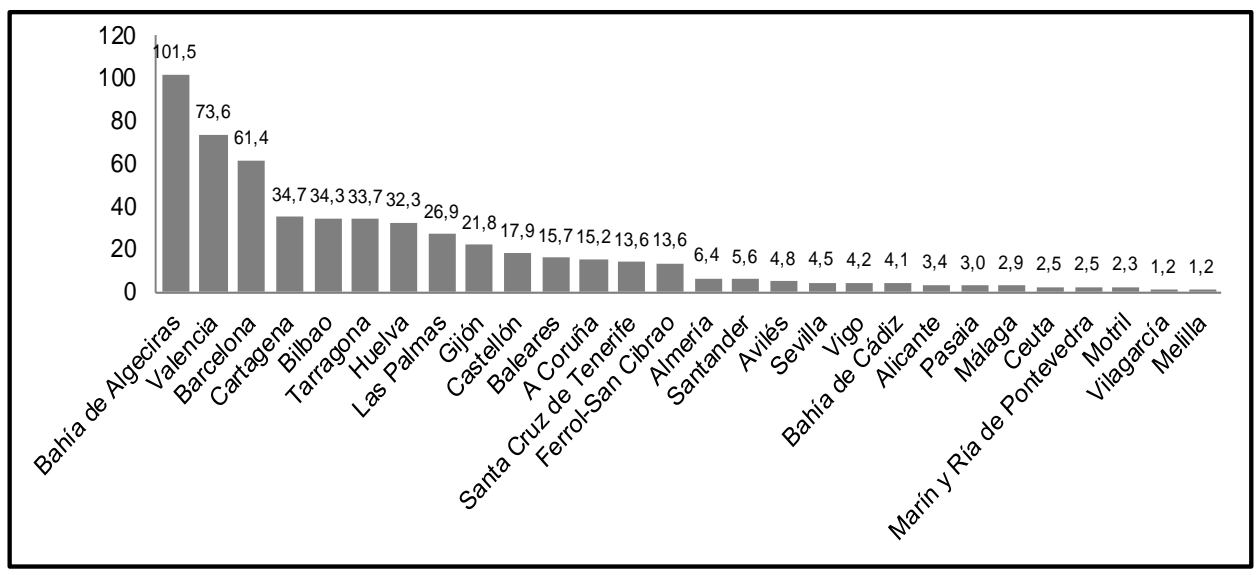

Fuente: Elaboración propia a partir de datos de las estadísticas de Puertos del Estado y de las memorias anuales de las Autoridades Portuarias.

En el tráfico de mercancías según su forma de presentación (graneles líquidos y sólidos, contenedores, mercancía general convencional) se movieron un total de 532 Mill. de Tm en 2017, lo que representan un 97,65\% sobre el total del tráfico portuario. En total se movieron 181 Mill. de Tm. de mercancías en contenedores, algo más de 178 Mill. de Tm. de graneles líquidos, 101 Mill. de Tm. de graneles sólidos y 71 Mill. de Tm. de mercancía general convencional. Los tres puertos con mayor tráfico de mercancías se encontraron en el Mediterráneo. El puerto Bahía de Algeciras consiguió mantenerse en primera posición tras lograr un movimiento de casi 97 Mill. de Tm., seguido por el de Valencia con más de 73 Mill. de Tm., en segunda posición, y el de Barcelona con 60 Mill. de Tm., en tercera posición.

Los graneles líquidos fueron las mercancías más voluminosas para la mayoría de las Autoridades Portuarias. El puerto de Bahía de Algeciras logró consolidar su primera posición tras alcanzar los 29 Mill. de Tm., con un incremento del 6,44\% en el año 2017. En segunda posición se situó el puerto de 
Cartagena que superó los 27 Mill. de Tm. con un incremento del 8,9\% y, en tercera posición, el puerto de Huelva que movió casi 25 Mill. de Tm., un 3,2\% más que en 2016. Por otra parte, hay que destacar que Pasaia fue el único puerto que no presentó ningún movimiento de graneles líquidos.

Figura 3

Ranking del tráfico de graneles de las 28 Autoridades Portuarias (en Mill. de Tm.). Año 2017.

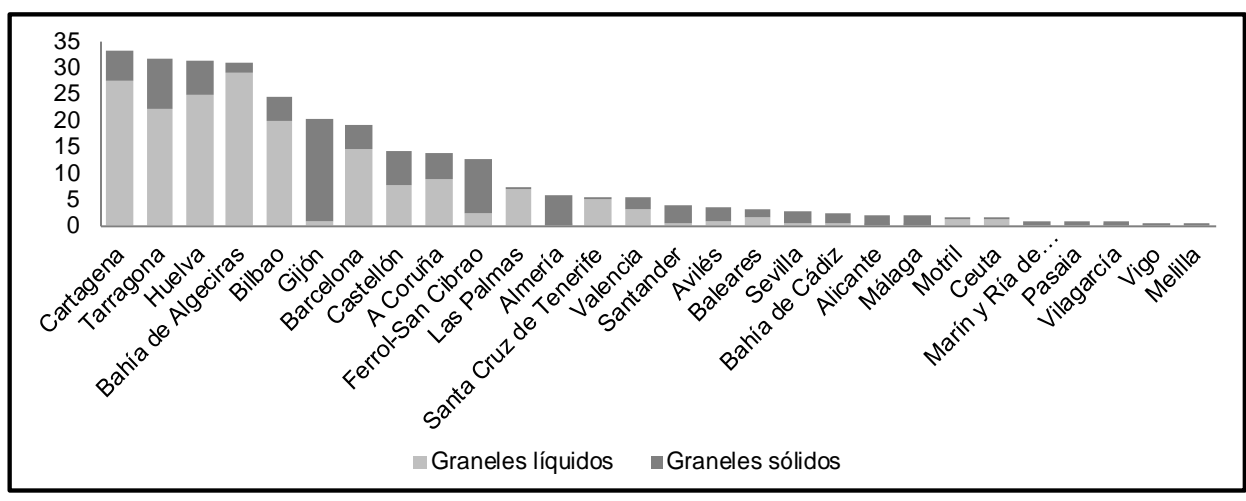

Fuente: Elaboración propia a partir de datos de las estadísticas de Puertos del Estado y de las memorias anuales de las Autoridades Portuarias

Además, la mayoría de las Autoridades Portuarias también experimentaron crecimientos en el tráfico de los graneles sólidos. Así pues, destacamos el puerto cántabro de Gijón por tener el mayor movimiento de graneles sólidos de todos, pues movió 19,2 Mill. de Tm. con una subida del 19,8\% en 2017, seguido del puerto de Ferrol-San Cibrao que alcanzó los 10,4 Mill. de Tm., con un crecimiento del 11,2\%, el de Tarragona que registró 9,5 Mill. de Tm. con un incremento menor del 5\%, y, por último, el de Huelva que movió casi 6,5 Mill. de Tm., con una subida del 12,6\% (Figura 3).

Por otra parte, el tráfico de pesca registró un crecimiento del 4,17\% en 2017 más que en el año anterior, siendo Vigo el primer puerto español en descargas de pesca fresca, superando los 89 Mill. de Tm. anuales (experimentando un incremento de 6,9\% en el año 2017), seguido del puerto de A Coruña con más de 51 Mill. (incremento de 16,9\%). La pesca arrojó resultados negativos para los puertos de Pasaia con 25,5 Mill. (reducción de 7,2\%) y el de Bahía de Cádiz con 16,3 Mill. (reducción de 6\%), aunque siguen manteniendo la tercera y cuarta posición. El de Avilés siguió en quinta posición y se recuperó un poco tras alcanzar 13,6 Mill. (incremento de 8,1\%), de acuerdo con las estadísticas publicadas por Puertos del Estado.

Por último, la llegada de pasajeros de cruceros a los 46 puertos de interés general a bordo de buques de cruceros está siguiendo una tendencia al alza 
(Figura 4). Según datos oficiales de Puertos del Estado, en los últimos doce años el tráfico de pasajeros de cruceros ha ido incrementando cada vez más hasta alcanzar en 2017 la cifra record de más de 9 Mill. de cruceristas, con un crecimiento del 6,7\%, y la llegada a los puertos españoles de 4.248 buques de cruceros, un 8,4\% más que en el año pasado. Los puertos de las Autoridades Portuarias de Barcelona y de las Islas Baleares (Palma, Alcúdia, Maó, Eivissa, La Savina) en el Mediterráneo son los destinos principales. Estos dos puertos acapararon el 63,7\% del total de cruceros en España con 7 Mill. de cruceristas en 2017. Las Autoridades Portuarias esperan superar estas cifras en los próximos años con la mejora de sus instalaciones para que puedan llegar buques más grandes de hasta $330 \mathrm{~m}$. de eslora y con más cruceristas a bordo.

Figura 4

Evolución del tráfico de cruceristas (en miles) y de buques de cruceros. Años 2005-2017

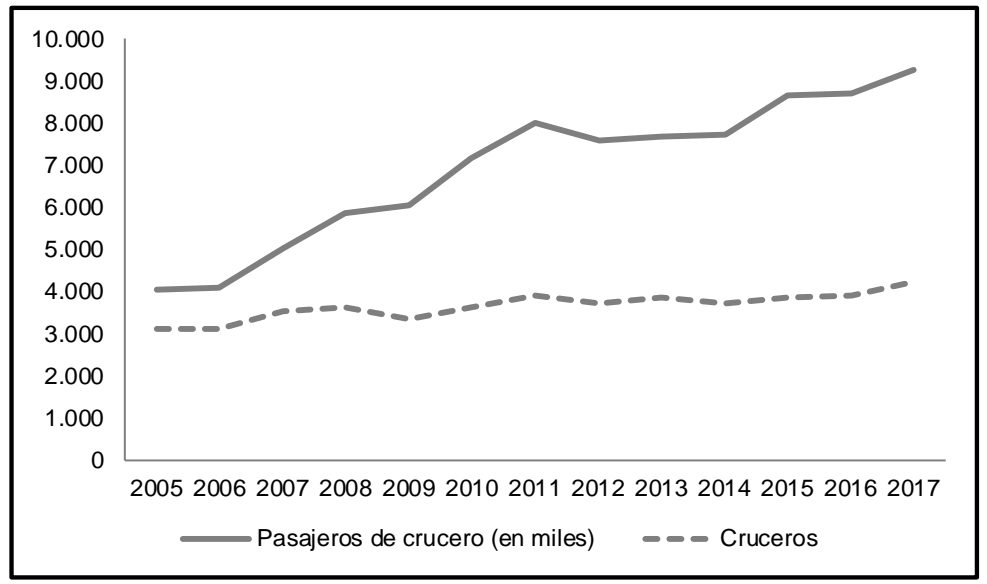

Fuente: Elaboración propia a partir de datos de las estadísticas de Puertos del Estado y de las memorias anuales de las Autoridades Portuarias.

\section{DESCRIPCIÓN DE LOS DATOS Y METODOLOGÍA}

Los datos económicos y de tráfico de las 28 Autoridades Portuarias necesarios para llevar a cabo este trabajo se han obtenido a partir de las memorias anuales de las propias Autoridades Portuarias y de las estadísticas mensuales y los anuarios anuales de Puertos del Estado, los cuales recogen información relevante de la actividad portuaria (Autoridades Portuarias, 2016; Puertos del Estado, 2016 y 2017).

En total se ha seleccionado un conjunto de dieciocho indicadores o variables cuantitativas relativas al volumen de tráficos portuarios y su especialización: mercancía en contenedores (exterior, en tránsito, embarcados, desembarcados, 
vacíos) y graneles tanto líquidos como sólidos, así como al tráfico de pasajeros de cruceros. Otros indicadores recogen las características de los puertos referentes a sus infraestructuras y servicios de que disponen, tales como: número de terminales de contenedores, longitud de las líneas de atraque; longitud de los muelles de contenedores, de graneles sólidos y de pasajeros; superficie de las terminales de contenedores y número de grúas de tipo portacontenedores y pórticos para la carga y descarga de contenedores de más de 16 toneladas. Por último, se han considerado dos indicadores cualitativos que hacen referencia a la disponibilidad de refinerías y de conexiones ferroviarias en los puertos.

En la Tabla 3 se muestra el nombre y la descripción de los 20 indicadores seleccionados, junto con los estadísticos descriptivos media y desviación típica de las dieciocho variables cuantitativas.

La metodología propuesta para la clasificación de los puertos españoles de interés general es el Análisis Factorial y el Análisis Clúster o de Conglomerados. Mediante el Análisis Factorial se consigue reducir el conjunto de variables iniciales. Esta técnica resulta especialmente adecuada cuando se dispone de un gran número de variables (Cuadras, 1981). Peneder (2005) presenta la aplicación del Análisis Clúster para la generación de clasificaciones industriales.

Para ver la idoneidad de la aplicación del Análisis Factorial de Componentes Principales se parte del examen de la matriz de correlaciones entre todas las variables, calculándose una serie de indicadores sobre la misma ${ }^{3}$. Asimismo, se llevan a cabo varios contrastes de hipótesis que evalúan si el modelo factorial en su conjunto es significativo. Uno de ellos es el Test de Esfericidad de Bartlett (Barlett, 1937) que contrasta la hipótesis nula de que la matriz de correlaciones se ajusta a la matriz identidad, lo que supone que las variables no están intercorrelacionadas y, por tanto, no tendría mucho sentido llevar a cabo un Análisis Factorial. Por otra parte, el índice KMO compara los coeficientes de correlación de Pearson con los coeficientes de correlación parcial entre variables y desaconseja el uso del Análisis Factorial cuando se obtienen valores bajos de este índice. Según Kaiser (1974), cuanto más próximo a uno esté el valor de este índice, más adecuado será la realización del procedimiento del Análisis Factorial.

De esta forma, se obtienen los factores, combinaciones lineales de las variables originales, que además están incorrelacionados entre sí. El método que se utiliza para su obtención nos garantiza que dichos factores estén ordenados en función a la información que explican que va a ser cuantificada a través de su varianza.

\footnotetext{
${ }^{3}$ Determinante de la matriz de correlaciones, matrices anti-imagen y medida de adecuación de la muestra
} 
Tabla 3

Descripción de las variables utilizadas

\begin{tabular}{|c|c|c|c|c|}
\hline Variable & Descripción & Unidad & Media & Desv. Típica \\
\hline FERROC & $\begin{array}{l}\text { Disponibilidad de conexiones } \\
\text { ferroviarias }\end{array}$ & $\begin{array}{l}1=\text { Sí, } \\
0=\text { No }\end{array}$ & - & - \\
\hline GRUAPORTACYPORT16 & $\begin{array}{l}\text { Total de grúas portacontenedores y } \\
\text { grúas pórticos para cargas y } \\
\text { descargas superiores a } 16 \\
\text { toneladas }\end{array}$ & $\mathrm{N}^{\circ}$ & 8,3 & 10,6 \\
\hline GTMEDBUPORTAC & $\begin{array}{l}\text { Arqueo medio de los buques } \\
\text { portacontenedores }\end{array}$ & GT & $15.092,9$ & $11.759,8$ \\
\hline LONGLINATRA & $\begin{array}{l}\text { Longitud de las líneas de atraque } \\
\text { de las terminales de contenedores }\end{array}$ & $\mathrm{m}$ & 894,8 & $1.234,2$ \\
\hline LONGMUCONT & $\begin{array}{l}\text { Longitud de los muelles de } \\
\text { contenedores }\end{array}$ & $\mathrm{m}$ & 852,9 & $1.279,4$ \\
\hline LONGMUGRSOL & $\begin{array}{l}\text { Longitud de los muelles de } \\
\text { graneles sólidos }\end{array}$ & $\mathrm{m}$ & $1.148,5$ & $1.287,1$ \\
\hline LONGMUPASAJ & $\begin{array}{l}\text { Longitud de los muelles de } \\
\text { pasajeros }\end{array}$ & $\mathrm{m}$ & $1.153,2$ & $1.469,8$ \\
\hline PASAJCRUC & Tráfico de pasajeros de cruceros & $\mathrm{N}^{\circ}$ & $310.495,4$ & $625.643,2$ \\
\hline PORCTRMERTUB & $\begin{array}{l}\text { Porcentaje de toneladas de tráfico } \\
\text { de mercancías por tuberías }\end{array}$ & $\%$ & 18,8 & 25,3 \\
\hline REFINER & Disponibilidad de refinerías & $\begin{array}{l}1=\text { Sí, } \\
0=\text { No }\end{array}$ & - & - \\
\hline SUPTERMCONT & $\begin{array}{l}\text { Superficie total de las terminales de } \\
\text { contenedores }\end{array}$ & $\mathrm{m}^{2}$ & $284.207,2$ & $495.562,0$ \\
\hline TERMCONT & Terminales de contenedores & $\mathrm{N}^{\circ}$ & 1,0 & 1,0 \\
\hline TEUDESEMB & Contenedores desembarcados & TEU & $271.284,8$ & $625.814,2$ \\
\hline TEUEMB & Contenedores embarcados & TEU & $269.940,3$ & $620.124,2$ \\
\hline TEUEXT & Tráfico de contenedores exterior & TEU & $188.297,3$ & $463.053,2$ \\
\hline TEUTRANS & Contenedores en tránsitos & TEU & $282.947,7$ & $919.582,3$ \\
\hline TEUVAC & Contenedores vacíos & TEU & $130.777,4$ & $260.729,4$ \\
\hline TRMERCONT & $\begin{array}{l}\text { Tráfico total de mercancía general } \\
\text { de contenedores }\end{array}$ & Tm & $6.010 .508,6$ & $14.775 .111,1$ \\
\hline TRMERGRLIQ & $\begin{array}{l}\text { Tráfico total de mercancia de } \\
\text { graneles líquidos }\end{array}$ & $\mathrm{Tm}$ & $5.985 .118,5$ & $8.572 .723,7$ \\
\hline TRMERGRSOL & $\begin{array}{l}\text { Tráfico total de mercancía de } \\
\text { graneles sólidos }\end{array}$ & $\mathrm{Tm}$ & $3.285 .480,3$ & $3.482 .390,9$ \\
\hline
\end{tabular}

Fuente: Elaboración propia a partir de datos de las estadísticas de Puertos del Estado y de las memorias anuales de las Autoridades Portuarias.

Los autovalores asociados a los factores obtenidos representan la contribución de cada factor a la variabilidad total de las variables. En esta línea, Kaiser (1974) propone elegir aquellas componentes principales cuyos autovalores sean mayores a la unidad. La rotación ortogonal denominada Varimax propuesta por Kaiser (1958) minimiza el número de variables que hay con pesos o saturaciones elevadas en cada factor. Además, la obtención de las componentes se realiza a través del procedimiento dado por Barttlet (1937), dado que éste diferencia mejor a los casos y es el más adecuado para la estimación de los factores comunes. 
Por otra parte, el Análisis Clúster o de Conglomerados es una técnica multivariante muy apropiada para realizar una clasificación de las 28 Autoridades Portuarias. Su finalidad es la clasificación de objetos formando grupos lo más homogéneos posible dentro de sí mismos y lo más heterogéneos posible entre sí. Son agrupamientos que descansan en la idea de distancia o similitud entre las observaciones, habiendo diversos criterios de medición de dichas distancias. Esta técnica multivariante, cuyos inicios se remontan a los trabajos de Sokal y Sneath (1963) se ha extendido en la actualidad a todos los ámbitos científicos.

El análisis de conglomerados jerárquico está indicado cuando no se conoce a priori el número de conglomerados y el número de casos no es excesivamente grande. El de tipo aglomerativo parte de tantos grupos como casos haya en la muestra y se van agrupando hasta conseguir un único clúster. Entre los criterios de agrupación más utilizados, el del vecino más lejano da lugar a conglomerados más compactos, siendo éste un criterio menos sensible a los outliers.

Finalmente, el número de conglomerados a obtener va a depender del lugar donde cortemos el dendograma, representación gráfica que muestra el proceso de agrupación jerárquica. Por ello, la decisión sobre el número de conglomerados más acertado va a depender principalmente de la interpretación que posteriormente pueda darse a cada uno de ellos, si bien el análisis de los coeficientes de distancia resulta igualmente útil en este proceso.

\section{RESULTADOS}

Con el propósito de clasificar los puertos españoles de titularidad estatal por medio de los indicadores o variables seleccionados atendiendo al tráfico, infraestructura y compromiso de los puertos tanto a nivel medioambiental como con la ciudad, se realiza, en primer lugar, un Análisis Factorial mediante el método de componentes principales para luego, a continuación, llevar a cabo el Análisis de Conglomerados, también denominado Análisis Clúster. El programa estadístico utilizado en la realización de todos los análisis ha sido el SPSS.

El procedimiento del análisis multivariante de tipo factorial permitió simplificar las múltiples relaciones que existen en el conjunto de las 16 variables utilizadas en un menor número de factores ortogonales, que explican suficientemente las variables originales y resultan ser fácilmente interpretables.

Para verificar la adecuación del Análisis Factorial, las variables cuantitativas originales deben estar altamente intercorrelacionadas, dado que si las correlaciones entre las variables son bajas el Análisis Factorial no sería apropiado. La Tabla 4 presenta algunos de los indicadores que nos permite comprobar el grado de asociación entre las variables originales.

En primer lugar, el valor tan bajo del determinante de la matriz de correlaciones de $6,306 \cdot 10^{-26}$, el cual es prácticamente igual a cero, indica la 
existencia de variables con relaciones lineales fuertes.

En segundo lugar, el índice KMO, que compara los coeficientes de correlación de Pearson con los coeficientes de correlación parcial entre variables, indica una adecuación de los datos a un modelo del Análisis Factorial ya que toma un valor de 0,77. Así, valores bajos del estadístico KMO desaconsejarían la utilización de este método del análisis multivariante.

En tercer lugar, para verificar si la matriz de correlaciones es o no una matriz identidad se ha aplicado el Test de esfericidad de Bartlett. El valor del estadístico de Bartlett de 1.131,502 y el nivel de significación p=0,000 nos lleva a rechazar la hipótesis nula que se trate de la matriz identidad, o lo que es lo mismo, la hipótesis nula de variables originales incorrelacionadas, por lo tanto, tiene sentido aplicar el Análisis Factorial.

Por último, los valores de las matrices anti-imagen quedaron muy bajos y la medida de adecuación a la muestra bastante alta en la diagonal de la matriz de correlaciones anti-imagen.

\section{Tabla 4}

Indicadores del grado de asociación entre las variables.

\begin{tabular}{|c|c|}
\hline Indicador & Coeficiente \\
\hline Determinante de la matriz de correlación & $6,306 * 10^{-26}$ \\
\hline Medida de adecuación muestral de Kaiser-Meyer-Olkin (KMO) & 0,770 \\
\hline $\begin{array}{r}\text { Chi-cuadrado aproximado } \\
\text { Grados de libertad } \\
\text { Significación }\end{array}$ & $\begin{array}{r}1.131,502 \\
190 \\
0,000\end{array}$ \\
\hline
\end{tabular}

Fuente: Elaboración propia.

Del Análisis Factorial se seleccionaron 4 factores incorrelados entre sí, que son combinación lineal de las 20 variables originales. La Tabla 5 recoge los autovalores asociados a los factores, los cuales representan la contribución de cada factor a la variabilidad total de las variables.

Tabla 5

Autovalores y porcentajes de varianza explicada por cada factor

\begin{tabular}{|c|c|c|c|}
\hline Factor & Autovalor & \% Varianza & \% Acumulado \\
\hline 1 & 10,810 & 54,050 & 54,050 \\
\hline 2 & 3,304 & 16,521 & 70,571 \\
\hline 3 & 1,798 & 8,991 & 79,562 \\
\hline 4 & 1,529 & 7,644 & 87,206 \\
\hline
\end{tabular}

Fuente: Elaboración propia.

Asimismo, con el objeto de obtener factores con una interpretación clara y relevante para la clasificación de los puertos españoles de titularidad estatal, y 
siguiendo a Kaiser (1974), se ha procedido a elegir aquellos factores con autovalores mayores a uno. De modo que, la varianza total explicada de estos cuatro primeros factores resume el $87,21 \%$ de la varianza total.

La Tabla 6 muestra la matriz de componentes rotados, la cual recoge la carga o ponderación de cada factor en cada una de las variables. El método de rotación utilizado ha sido el Varimax, que consiste en una rotación ortogonal en la que la rotación de los ejes asegura la incorrelación entre los factores. El procedimiento de rotación ha permitido mejorar la capacidad de interpretación de los factores, dado que cada variable tiene correlaciones próximas a uno con alguno de los factores y próximas a cero con el resto.

Tabla 6

Matriz de componentes rotados

\begin{tabular}{|c|c|c|c|c|}
\hline Variable & Factor 1 & Factor 2 & Factor 3 & Factor 4 \\
\hline TEUDESEMB & ,984 & 081 &, 029 &,- 056 \\
\hline TEUEMB & ,984 & 084 & 028 &,- 055 \\
\hline TRMERCONT & ,974 & , 109 &,- 045 &,- 075 \\
\hline TEUVAC & ,969 &,- 010 & 196 & ,011 \\
\hline SUPTERMCONT & ,936 &,- 084 & ,228 & ,090 \\
\hline LONGMUCONT & 921 & 089 & ,268 & ,022 \\
\hline GRUAPORTACYPORT16 & 913 & ,015 & ,219 & 222 \\
\hline LONGLINATRA & 899 & 025 & 342 & 081 \\
\hline TEUTRANS & 890 & 171 &,- 210 &,- 181 \\
\hline GTMEDBUQPORTAC & ,858 & 022 & ,106 & 093 \\
\hline TEUEXT & ,805 &,- 105 & ,333 & ,237 \\
\hline TERMCONT & ,711 & ,069 & ,445 & ,235 \\
\hline REFINER & ,055 & ,950 &,- 081 & , 155 \\
\hline PORCTRMERTUB &,- 166 & ,919 & ,039 & ,153 \\
\hline TRMERGRLIQ & ,301 & ,876 &,- 009 & ,204 \\
\hline PASAJCRUC & ,191 &,- 059 & ,940 &,- 049 \\
\hline LONGMUPASAJ & ,323 &, 015 & ,859 &,- 226 \\
\hline LONGMUGRSOL & ,045 & ,211 & ,033 & ,845 \\
\hline TRMERGRSOL &,- 089 & , 108 &,- 074 & ,828 \\
\hline FERROC & 292 & 235 &,- 280 & ,559 \\
\hline
\end{tabular}

Fuente: Elaboración propia.

Si atendemos a las características de las variables que están asociadas a cada uno de los cuatro factores, se pueden encontrar rasgos comunes que nos permiten identificar el factor correspondiente y poder darle una denominación. De este modo, el "Factor 1" explica por sí solo el 54,05\% de la varianza total y se encuentra identificado con doce variables relacionadas con el "Tráfico de contenedores", que son: GRUAPORTACYPORT16, GTMEDBUPORTAC, LONGLINATRA, LONGMUCONT, SUPTERMCONT, TERMCONT, TEUDESEMB, TEUEMB, TEUEXT, TEUTRANS, TEUVAC y TRMERCONT. En 
este sentido, cabe señalar que el tráfico de contenedores en tránsito (TEUTRANS) en los puertos españoles está condicionado tanto por la actividad económica a nivel mundial como por la posición de nuestras terminales portuarias en relación con las grandes rutas internacionales, la ruta Este-Oeste-Este que atraviesa los estrechos de Malaca, Suez, Gibraltar y Panamá y la ruta Norte-Sur entre Europa y África y Europa América del Sur.

Tabla 7

Puntuaciones factoriales

\begin{tabular}{|c|c|c|c|c|}
\hline Autoridades Portuarias & Factor 1 & Factor 2 & Factor 3 & Factor 4 \\
\hline A Coruña & $-0,5285$ & 1,2542 & $-0,2197$ & $-0,0802$ \\
\hline Alicante & $-0,22230$ & $-0,6623$ & $-0,3766$ & $-0,0547$ \\
\hline Almería & $-0,5263$ & $-0,7235$ & $-0,4548$ & $-0,1257$ \\
\hline Avilés & $-0,4966$ & $-0,4570$ & $-0,4442$ & $-0,6669$ \\
\hline Bahía de Algeciras & 2,9400 & 1,6302 & $-1,6491$ & $-1,3130$ \\
\hline Bahía de Cádiz & $-0,2835$ & $-0,5189$ & 0,0507 & $-0,3511$ \\
\hline Baleares & $-0,8677$ & $-0,0746$ & 2,4673 & $-0,9906$ \\
\hline Barcelona & 1,3893 & $-0,4808$ & 3,0866 & 1,0446 \\
\hline Bilbao & 0,0415 & 1,1805 & 0,1603 & 1,6159 \\
\hline Cartagena & $-0,4642$ & 2,0222 & 0,0436 & 0,6220 \\
\hline Castellón & $-0,1759$ & 0,9942 & $-0,1795$ & 0,4770 \\
\hline Ceuta & $-0,4737$ & $-0,5069$ & $-0,2883$ & $-1,1373$ \\
\hline Ferrol-San Cibrao & $-0,2940$ & $-0,8228$ & $-0,3490$ & 0,4449 \\
\hline Gijón & $-0,3704$ & $-1,1581$ & $-0,4368$ & 3,1093 \\
\hline Huelva & $-0,5550$ & 1,9996 & $-0,5082$ & 0,0254 \\
\hline Las Palmas & 0,4861 & $-0,1806$ & 1,6484 & $-0,6618$ \\
\hline Málaga & $-0,1865$ & $-0,6267$ & 0,3680 & $-0,7723$ \\
\hline Marín y Ría de Pontevedra & $-0,0758$ & $-0,7270$ & $-0,6710$ & $-0,0243$ \\
\hline Melilla & $-0,4176$ & $-0,5711$ & $-0,4088$ & $-1,0625$ \\
\hline Motril & $-0,5688$ & 0,1056 & $-0,2091$ & $-1,1248$ \\
\hline Pasaia & $-0,3603$ & $-0,6465$ & $-0,8567$ & $-0,3887$ \\
\hline Santa Cruz de Tenerife & $-0,2962$ & 1,2186 & 1,3744 & $-1,0636$ \\
\hline Santander & $-0,4235$ & $-0,7481$ & $-0,7621$ & 0,6462 \\
\hline Sevilla & $-0,2273$ & $-0,6955$ & $-0,4208$ & 0,2885 \\
\hline Tarragona & $-0,2779$ & 1,5009 & $-0,0269$ & 1,6397 \\
\hline Valencia & 3,5187 & $-1,0069$ & $-0,0577$ & 0,4099 \\
\hline Vigo & $-0,0559$ & $-0,6414$ & $-0,2533$ & $-0,3709$ \\
\hline Vilagarcía & $-0,2271$ & $-0,6575$ & $-0,6268$ & $-0,1349$ \\
\hline
\end{tabular}

Fuente: Elaboración propia.

El "Factor 2" viene denominado como "Tráfico de graneles líquidos y refinerías" y explica el $16,52 \%$ de la varianza total con las tres variables: PORCTRMERTUB, REFINER y TRMERGRLIQ. El "Factor 3" explica el $8,99 \%$ de la varianza total y está relacionado con el "Tráfico de pasajeros de cruceros" con las siguientes dos variables: LONGMUPASAJ y PASAJCRUC. Por último, el "Factor 4" viene denominado como "Tráfico de graneles sólidos" 
y explica el 7,64\% de la varianza total con las tres variables: FERROC, LONGMUGRSOL y TRMERGRSOL.

En cuanto a la obtención de la matriz de puntuaciones factoriales, el procedimiento utilizado ha sido el método de Barttlet (1937), dado que éste diferencia mejor a los puertos españoles de titularidad estatal y es el más adecuado para la estimación de los factores comunes.

En la Tabla 7 se recogen las puntuaciones factoriales. En primer lugar, los puertos de Valencia $(3,52)$ y Bahía de Algeciras $(2,94)$ muestran puntuaciones factoriales elevadas en el "Factor 1" de tráfico de contenedores. En segundo lugar, los puertos de Cartagena $(2,02)$, Huelva $(1,99)$, Bahía de Algeciras $(1,63)$ y Tarragona $(1,50)$ obtienen puntuaciones factoriales muy altas en el "Factor 2" de tráfico de graneles líquidos y refinerías. Sin duda, la presencia de refinerías en estos puertos constituye una fuente de ingresos y suponen el motor para el desarrollo del tráfico de graneles líquidos en estos puertos.

En tercer lugar, los puertos de Barcelona $(3,09)$ y Baleares $(2,47)$ muestran las puntuaciones factoriales más elevadas en el "Factor 3" de tráfico de pasajeros de cruceros. A continuación, le siguen los puertos canarios de Las Palmas $(1,65)$ y Santa Cruz de Tenerife $(1,37)$ que también tienen puntuaciones factoriales altas en este factor. Por último, los puertos de Gijón $(3,11)$, Tarragona $(1,64)$ y Bilbao $(1,62)$ presentan las puntuaciones más altas en el "Factor 4" de tráfico de graneles sólidos. De hecho, mientras que el puerto de Gijón está dedicado especialmente al tráfico de graneles sólidos, los puertos de Tarragona y Bilbao se dedican tanto a los graneles líquidos como a los sólidos.

A continuación, se muestran los resultados obtenidos con el Análisis de Conglomerados o Clúster de tipo jerárquico, que nos permite la clasificación de los puertos en grupos lo más homogéneos posibles utilizando ciertas variables, las cuales son en este caso, los cuatro factores obtenidos en el Análisis Factorial previo. Además, se ha utilizado la distancia euclídea al cuadrado como medida de distancia más apropiada para los datos cuantitativos analizados, y "el vecino más lejano” como criterio de agrupación, el cual determina la distancia entre dos grupos a partir de la distancia de los dos puntos más alejados.

En el dendograma de la Figura 5, los grupos se configuran con estructura arborescente, es decir, los clústeres de niveles más bajos se van englobando en otro de niveles superiores. Esta representación gráfica se ha utilizado para evaluar la cohesión de los conglomerados que se han formado y proporcionar información sobre el número adecuado de conglomerados.

Así, teniendo en cuenta el dendograma, se pueden distinguir cuatro grupos claramente diferenciados en la Tabla 8. En el primer grupo se encuentran los dos puertos de transbordos o hubs de Bahía de Algeciras y Valencia, los cuales se caracterizan por ser los puertos españoles de titularidad estatal con mayores volúmenes de tráfico portuario y mayores relevancias en el tráfico internacional, 
llegando a alcanzar los 101,5 y 73,6 Mill. de Tm. en el año 2017, respectivamente (véase Figura 2). Estos puertos son los que presentan las mayores puntuaciones factoriales en el "Factor 1", con un tráfico de contenedores de más de 4,38 y 4,83 Mill. de TEUs, respectivamente, y con alrededor de un $92 \%$ y 53\% de contenedores en modalidad de tránsito, respectivamente. El arqueo medio de los buques portacontenedores supera los 44.000 GT y la longitud de las líneas de atraque en las terminales de contenedores es mayor a los 3.000 metros en ambos puertos.

Figura 5

Dendograma

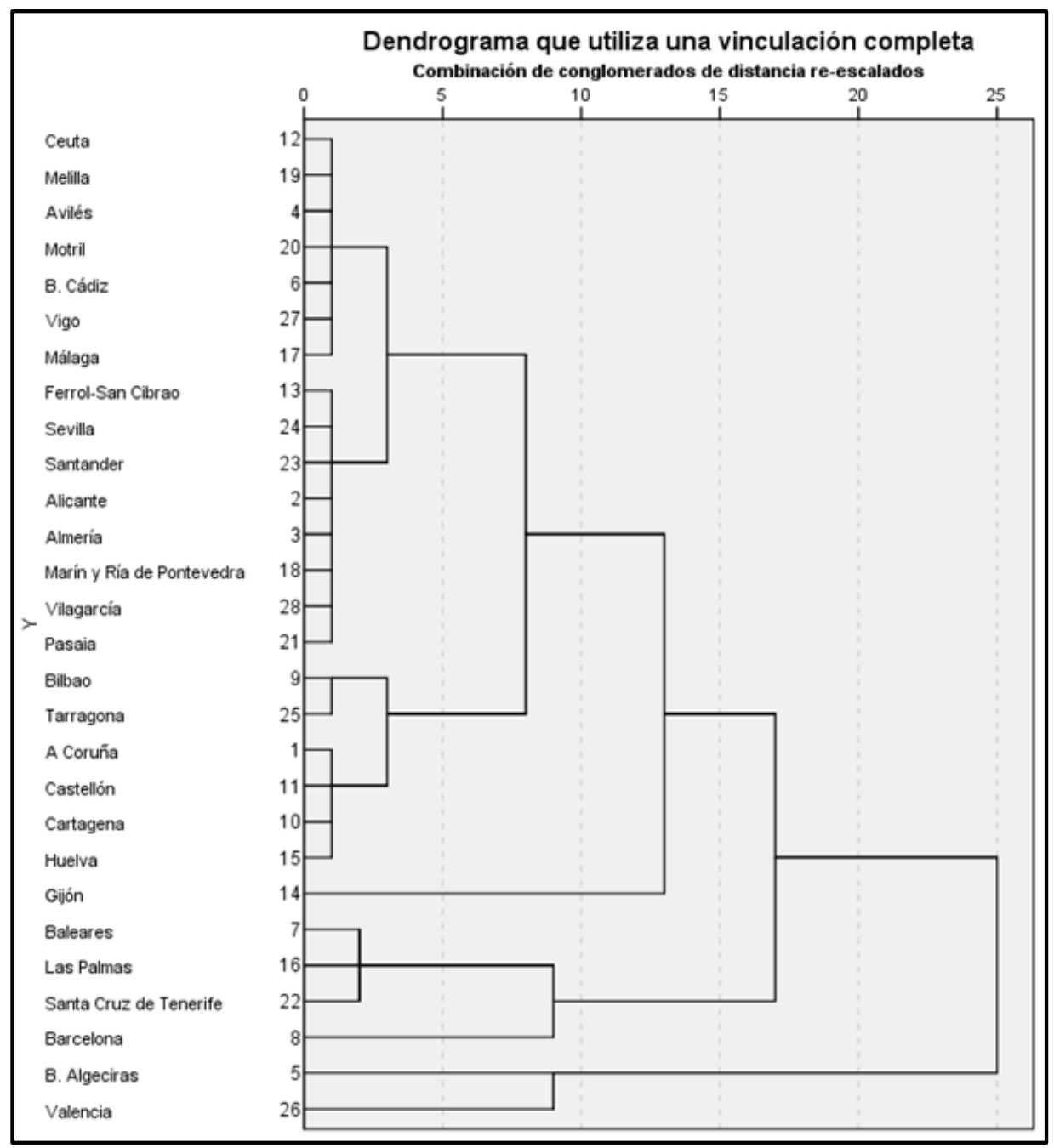

Fuente: Elaboración propia 
En el segundo grupo se recogen los cuatro puertos que presentan mayores puntuaciones factoriales en el "Factor 3" de tráfico de pasajeros de cruceros. A este grupo pertenecen los puertos mediterráneos de Barcelona y Baleares, que son los que presentan un mayor tráfico de pasajeros, ya que movieron 2,72 y 2,13 Mill. de personas en 2017, respectivamente, y los puertos canarios de Las Palmas y Santa Cruz de Tenerife con 1,23 y 0,96 Mill. de personas, respectivamente. El puerto de Barcelona también se caracteriza por ser un puerto hub de hidrocarburos que cuenta con 9 terminales, de las cuales 7 son terminales de cruceros. Además, su ubicación geográfica y la conexión con la red de ferrocarril europea lo convierten en una infraestructura con futuro. Por su parte, el puerto hub de Las Palmas sirve de base logística para mercancías de África, Europa y América y, además, es la principal entrada de mercancías para la Comunidad.

De acuerdo con Castillo-Manzano et al. (2014), entre los factores que afectan la capacidad de los puertos para atraer cruceros en España se encuentran los siguientes: que los puertos estén ubicados en áreas pobladas y cerca de grandes aeropuertos; y que no estén especializados en el tráfico de contenedores, pero que compartan instalaciones con tráfico de ferris. Así, el tráfico de cruceros que puede generar un puerto también está relacionado con el atractivo turístico y las instalaciones compartidas con otros tipos de tráficos portuarios, como son: el tráfico por medios rodados roll-on/roll-off (ro-ro) y los ferris.

El tercer grupo lo forma un único puerto, el de Gijón, que se caracteriza por ser el principal puerto español en tráfico de mercancías de graneles sólidos, alcanzando unos 19,2 Mill. de Tm. en el año 2017 con altas puntuaciones factoriales en el "Factor 4" de tráfico de graneles sólidos. Este puerto destina 9 de sus terminales al transporte de este tipo de mercancías y está posicionado como un referente del Arco Atlántico.

Por último, el cuarto grupo es el que concentra la mayoría de los puertos, un total de 21. Es en realidad un grupo mixto en el que se recogen cuatro subgrupos diferenciados. El primer subgrupo lo forman los puertos de A Coruña, Castellón, Cartagena y Huelva, que presentan altas puntuaciones factoriales en el "Factor 2" de tráfico de graneles líquidos y refinerías. Entre los cuatro puertos se movieron 68,8 Mill. de Tm. en 2017, lo que representa el 38,52\% del total del volumen de tráficos en mercancías de graneles líquidos. Son puertos que cuentan con acceso ferroviario y una refinería de petróleo en sus inmediaciones. Además, el puerto de Castellón realiza la función de centro secundario del arco mediterráneo, descongestionando tráfico de Tarragona y Valencia y siendo lugar de entrada y salida para productos de cerámica. En el segundo subgrupo se recogen los puertos de Bilbao y Tarragona, que presentan altas puntuaciones factoriales tanto en el "Factor 2" como en el "Factor 4", y tienen instalaciones ferroviarias, oleoductos y refinerías de petróleo ubicadas en el entorno portuario. Bilbao es el puerto de referencia de carga para la costa del Cantábrico y puerta de entrada y 
salida de mercancías a la Meseta, además cuenta con una planta regasificadora y un gasoducto que permite la circulación del gas con Francia y la interconexión entre ambos países. Por su parte, el puerto de Tarragona destaca por ser un puerto hub de gases energéticos del petróleo.

Tabla 8

Agrupamiento según el dendograma

\begin{tabular}{|c|c|c|}
\hline Grupo & Subgrupo & Autoridades Portuarias incluidas \\
\hline 1 & & $\begin{array}{l}\text { - Bahía de Algeciras } \\
\text { - Valencia }\end{array}$ \\
\hline 2 & & $\begin{array}{l}\text { - } \text { Baleares } \\
\text { - Las Palmas } \\
\text { - Santa Cruz de Tenerife } \\
\text { - Barcelona }\end{array}$ \\
\hline 3 & & - Gijón \\
\hline \multirow{4}{*}{4} & 4.1 & $\begin{array}{l}\text { - A Coruña } \\
\text { - Castellón } \\
\text { - Cartagena } \\
\text { - Huelva } \\
\end{array}$ \\
\hline & 4.2 & $\begin{array}{ll}\text { - } & \text { Bilbao } \\
\text { - } & \text { Tarragona }\end{array}$ \\
\hline & 4.3 & $\begin{array}{l}\text { - Ferrol-San Cibrao } \\
\text { - Sevilla } \\
\text { - Santander } \\
\text { - Alicante } \\
\text { - Almería } \\
\text { - Marín y Ría de Pontevedra } \\
\text { - Vilagarcía } \\
\text { - Pasaia }\end{array}$ \\
\hline & 4.4 & $\begin{array}{l}\text { - } \text { Ceuta } \\
\text { - Melilla } \\
\text { - Avilés } \\
\text { - Motril } \\
\text { - Bahía de Cádiz } \\
\text { - Vigo } \\
\text { - Málaga }\end{array}$ \\
\hline
\end{tabular}

Fuente: Elaboración propia.

El tercer y cuarto subgrupo se corresponde con quince puertos que no poseen refinerías de petróleo en sus inmediaciones y que presentan el menor volumen de tráfico de mercancías, en concreto un volumen inferior a los 13,5 Mill. de Tm. Así, mientras el tercer subgrupo lo forman los puertos de Ferrol-San Cibrao, Sevilla, Santander, Alicante, Almería, Marín y Ría de Pontevedra, Vilagarcía y Pasaia, en el cuarto subgrupo se encuentran los puertos de Ceuta, Melilla, Avilés, Motril, Bahía de Cádiz, Vigo y Málaga. De todos ellos, los puertos de Almería y Ferrol-San Cibrao son los dos únicos puertos que no son públicos. Por otra parte, los puertos de Vigo y Pasaia destacan porque son los que presentan un mayor 
volumen de tráfico portuario de pesca, con 89 y 25,6 miles de Tm. en el año 2017, respectivamente.

\section{CONCLUSIONES}

Dada la relevancia del sector de transporte marítimo en España, la cual está impulsada por su excelente situación geográfica en el extremo suroccidental de Europa, con un amplio litoral que da salida tanto al océano Atlántico como al mar Mediterráneo, y próxima a los ejes de las rutas marítimas internacionales más importantes, el presente estudio ha realizado una importante clasificación de los puertos españoles de interés general del Estado por medio de dos técnicas del análisis multivariante, el Análisis Factorial de Componentes Principales y el Análisis de Conglomerados.

De hecho, los puertos españoles son conscientes de que constituyen la principal plataforma de desarrollo de las ciudades y de su entorno. Asimismo, en los últimos años los puertos españoles están llevando a cabo diversas acciones, tanto de transformación física de su espacio portuario como medioambientales de conservación y mejora de la explotación portuaria, así como también acciones de fomento de la transparencia y de conocimiento del puerto por la ciudadanía.

Como conclusiones relevantes del análisis presentado en este trabajo cabe resaltar la existencia de cuatro grupos de puertos de titularidad estatal claramente diferenciados, tres de ellos en función de diversos indicadores que caracterizan la actividad portuaria referentes al volumen del tráfico portuario y especialización de mercancías (contenedores, graneles líquidos, graneles sólidos), infraestructuras y servicios portuarios de que disponen, y otro grupo de puertos que destacan por el tráfico de pasajeros de cruceros.

Los puertos de Bahía de Algeciras y Valencia son los puertos españoles de titularidad estatal con mayor tráfico portuario y a su vez, son también los más relevantes en el tráfico internacional. Cabe destacar sus altos niveles de tráfico de contenedores en modalidad de tránsito; en concreto, en Bahía de Algeciras, el 92\% de los contenedores lo son en tránsito. Esta situación viene explicada por la localización geográfica de sus terminales portuarias, que favorece su inclusión en las grandes rutas internacionales.

Otro grupo significativo, es el formado por los puertos de Barcelona, Baleares, y los puertos canarios de Las Palmas y Santa Cruz, que localizados en ciudades con gran interés turístico destacan por su mayor tráfico de pasajeros de cruceros.

El tercer grupo incluye únicamente al puerto de Gijón, que se distingue por ser el principal puerto español en tráfico de mercancías de graneles sólidos y que es considerado todo un referente del Arco Atlántico.

Por último, el cuarto grupo es el que aglutina la mayoría de los puertos. Es un conglomerado en el que se recogen cuatro subgrupos diferenciados. El primer 
subgrupo lo forman los puertos de A Coruña, Castellón, Cartagena y Huelva, puertos que cuentan con acceso ferroviario y una refinería de petróleo cercana, que demanda altos volúmenes de tráfico de graneles líquidos. El segundo subgrupo incluye los puertos de Bilbao y Tarragona, que poseen instalaciones ferroviarias, oleoductos y refinerías de petróleo situadas en el entorno portuario. En este sentido, el puerto de Tarragona destaca por ser un puerto hub de gases energéticos del petróleo. Finalmente, los quince puertos incluidos en los subgrupos tercero y cuarto son puertos de menor envergadura en relación al tráfico de mercancías, no superando ninguno de ellos los 13,5 Mill. de Tm.

En este contexto, y dado que el transporte marítimo es el principal modo utilizado para el comercio internacional de mercancías, representando el 80\% de esta actividad, los puertos como nodos logísticos e intermodales requieren un especial estudio y clasificación, para poder aplicar políticas adecuadas que permitan optimizar su gestión.

\section{REFERENCIAS BIBLIOGRÁFICAS}

AUTORIDADES PORTUARIAS (2016). "Memorias de las Autoridades Portuarias y Anuario Estadístico de Puertos del Estado 2016". Disponible en: http://www.puertos.es/Memorias_Anuales/2016/index.html [Último acceso: Julio de 2018].

BAÑOS-PINO, J.; COTO-MILLÁN, P. y RODRÍGUEZ-ÁLVAREZ, A. (1999). "Allocative Efficiency and Over-capitalization: An Application". International Journal of Transport Economics, 26(2), pp. 181-199.

BARLETT, M.S. (1937). "The Statistical Conception of Mental Factors". British Journal of Psychology, 28(1), pp. 97-104.

CAMARERO ORIVE, A. y CAMARERO ORIVE, A. (2016). Propuesta Metodológica para la clasificación de Puerto Verde mediante el análisis de Conglomerados y la utilización de Indicadores de Sostenibilidad Medioambientales. Brasil: IIICIDESPORT Congreso Internacional de Desempenhto Portuario.

CAMARERO ORIVE, A.; CERBÁN JIMÉNEZ, M.M.; TURIAS DOMÍNGUEZ, I.J.; GONZÁLEZ CANCELAS, N. y CAMARERO ORIVE, A. (2016). "Clasificación de los Puertos Españoles mediante Indicadores de Explotación utilizando Análisis de Conglomerados". INGE CUC, 12(2), pp. 41-49.

CASTILLO-MANZANO, J.I.; FAGEDA, X. y GONZÁLEZ-LAXE, F. (2014). "An Analysis of the Determinants of Cruise Traffic: An Empirical Application to the Spanish Port System". Transportation Research Part E: Logistics and Transportation Review, 66(C), pp. 115-125.

CE (1978). "Constitución Española". Boletín Oficial del Estado, 29 de diciembre de 1978, núm. 311, pp. 29313-29424.

CUADRAS, C.M. (1981). Métodos de Análisis Multivariante. Barcelona: EUNIBAR.

GARCÍA DEL HOYO, J.J.; CORDÓN LAGARES, E. y CORTÉS RODRÍGUEZ, C. (2001). La Infraestructura Portuaria Disponible como Factor Determinante de la Estructura del 
Sector Pesquero Andaluz. A Coruña: XXV Congreso Internacional de Economía Aplicada ASEPELT.

GONZÁLEZ, M.M. y TRUJILLO, L. (2003). Análisis de la Eficiencia de los Servicios de Infraestructura en España: Una Aplicación al Tráfico de Contenedores. Santa Cruz de Tenerife: X Encuentro de Economía Pública.

GONZÁLEZ, M.M. y TRUJILLO, L. (2009). "Efficiency Measurement in the Port Industry: A Survey of the Empirical Evidence". Journal of Transport Economics and Policy, 43(2), pp. 157-192.

GONZÁLEZ LAXE, F.; FREIRE SEOANE, M.J. y PAIS MONTES, C. (2015). "Política y Evaluación de la Selección Portuaria: El Caso Español". Revista de Evaluación de Programas y Políticas Públicas, 4, pp. 82-102.

IGN (2018): "Datos Geográficos y Toponimia: Longitud de la Línea de Costa Española por Provincias". Disponible en: http://www.ign.es/web/ign/portal/ane-datos-geograficos//datos-geograficos/datosGenerales?tipoBusqueda=longCosta [Último acceso: Julio de 2018].

KAISER, H. (1958). "The Varimax Criterion for Analytic Rotation in Factor Analysis". Psychometrika, 23, pp. 187-200.

KAISER, H. (1974). "An Index of Factorial Simplicity". Psychometrika, 39, pp. 31-36.

LEY 27/1992, de 24 de noviembre, de Puertos del Estado y de la Marina Mercante. Boletín Oficial del Estado, 25 de noviembre de 1992, núm. 283, pp. 39953-39984.

LEY DE PUERTOS, de 7 de mayo de 1880. Gaceta de Madrid, 8 de mayo de 1880, núm. 129, pp. 331-333. Disponible en: https://www.fundacionmapfre.org/documentacion/ publico/i18n/catalogo_imagenes/grupo.cmd?path=1003926 [Último acceso: Julio de 2018].

OMC (2018). "Informe Anual 2018". Disponible en: https://www.wto.org/spanish/res_s/ publications_s/anrep18_s.htm [Último acceso: Julio de 2018].

PENEDER (2005). "Creating Industry Classifications by Statistical Cluster Analysis". Estudios de Economía Aplicada 23-2, pp. 451-463.

PORTECONOMICS (2018). "PortGraphic: The Top 15 Container Ports in Europe in 2017". Disponible en: http://www.porteconomics.eu/2018/02/28/portgraphic-the-top-15container-ports-in-europe-in-2017/ [último acceso: Julio de 2018].

PUERTOS DEL ESTADO (2016). "Anuario Estadístico del Sistema Portuario de Titularidad Estatal 2016". Disponible en: http://www.puertos.es/es-es/estadisticas/RestoEstad \%c3\%adsticas/anuariosestadisticos/Paginas/2016.aspx [Último acceso: Julio de 2018].

PUERTOS DEL ESTADO (2017). "Estadística Mensual 2017". Disponible en: http://www.puertos.es/es-es/estadisticas/Paginas/estadistica_mensual.aspx. [Último acceso: Julio de 2018].

PUERTOS DEL ESTADO (2018). "Información Institucional". Disponible en: http://www.puertos.es/es-es/nosotrospuertos/Paginas/Nosotros.aspx [Último acceso: Julio de 2018].

REAL DECRETO LEGISLATIVO 2/2011, de 5 de septiembre, por el que se aprueba el Texto Refundido de la Ley de Puertos del Estado y de la Marina Mercante. Boletín Oficial del Estado, 20 de octubre de 2011, núm. 253, pp. 109456-109710.

SOKAL, R. y SNEATH, P.H. (1963). Principles of Numerical Taxonomy. San Francisco: W.H. Freeman and Co.

UNCTAD (2017). Review of Maritime Transport 2017. Nueva York y Geneva: Naciones Unidas para el Comercio y el Desarrollo. 
788 C. Cortés Rodríguez, E. Cordón Lagares, A. GonzÁlez Galán y J. J. García del Hoyo

WANHILL, S.R.C. (1974). "A Study in Port Planning: The Example of Mina Zayed". Maritime Policy and Management, 2(1), pp. 48-55. 